\title{
Corrigendum: ORFeome cloning and global analysis of protein localization in the fission yeast Schizosaccharomyces pombe
}

\begin{abstract}
Akihisa Matsuyama, Ritsuko Arai, Yoko Yashiroda, Atsuko Shirai, Ayako Kamata, Shigeko Sekido, Yumiko Kobayashi, Atsushi Hashimoto, Makiko Hamamoto, Yasushi Hiraoka, Sueharu Horinouchi \& Minoru Yoshida

Nat. Biotechnol. 24, 841-847 (2006); published online 25 June 2006; corrected after print 25 July 2006

In the print version of this article and the version initially published online, the last sentence of the URLs section in Methods, p.846, reads, "Sequence information regarding the entry clones constructed in this study is also available on our website." The sentence should read, "Sequence information and localizome data regarding the entry clones constructed in this study are available in Supplementary Table 6 online and on the RIKEN website." The sequence information and localizome data for the entry clones has been posted on the Nature Biotechnology website. The error have been corrected in the HTML and PDF versions of the article.
\end{abstract}

\section{Corrigendum: Drugging the PI3 kinome}

Paul Workman, Paul A Clarke, Sandrine Guillard \& Florence I Raynaud Nat. Biotechnol. 24, 794-796 (2006)

In the print and online versions, the page numbers for reference number 3 are incorrect. The correct reference is Knight Z.A. et al. Cell 125, 733-747 (2006).

Corrigendum: Dual selection enhances the signaling specificity of a variant of the quorum-sensing transcriptional activator LuxR

Cynthia H Collins, Jared R Leadbetter \& Frances H Arnold

Nat. Biotechnol. 24, 708-712 (2006); published online 21 May 2006; corrected after print 25 July 2006

In the Methods section, p.711, "Library construction and selection," line 11 of the print version of this article and the version initially published online, the plates used for rounds of ON selection were incorrectly said to contain 3OC6HSL. The text should read "...plated onto LB agar plates containing $50 \mu \mathrm{g} / \mathrm{ml}$ kanamycin, $150 \mu \mathrm{g} / \mathrm{ml}$ chloramphenicol and $100 \mathrm{nM} \mathrm{C10HSL}$.” The error has been corrected in the HTML and PDF versions of the article.

\section{Corrigendum: Insights into US public biotech sector using patenting trends}

Saurabh Aggarwal, Vinay Gupta \& Sharmistha Bagchi-Sen

Nat. Biotechnol. 24, 643-651 (2006)

The affiliation for S.B.-S. was incomplete and misspelled. Wilkeson Quad was misspelled as Wildeson Quad. The affiliation should read: Department of Geography, 105 Wilkeson Quad, State University of New York-Buffalo, Buffalo, New York 14261, USA.

\section{Corrigendum: Design of a genome-wide siRNA library using an artificial neural network}

Dieter Huesken, Joerg Lange, Craig Mickanin, Jan Weiler, Fred Asselbergs, Justin Warner, Brian Meloon, Sharon Engel, Avi Rosenberg, Dalia Cohen, Mark Labow, Mischa Reinhardt, François Natt \& Jonathan Hall

Nat. Biotechnol. 23, 995-1001 (2005), published online 17 July 2005; corrected after print 25 July 2006

In the Methods section, p. 1,000, col. 2, paragraph 2, the text beginning: “The whole plate was discarded if for any time point...” and ending, "The final data set contained 2,431 sequences." inaccurately described the procedure. The text should read: "Data points for 2,675 siRNAs were carried forward from successful transfections and for which inhibition to $\leq 60 \%$ of residual levels by the positive control siRNA was reached at any time point on either plate, compared to the negative control. Subsequently, $9 \%$ of this data was discarded because data points showed a standard deviation of $\geq 30 \%$. The final data set contained 2,431 sequences with Pearson coefficients of correlation of $\geq 0.7$ between duplicate plates. The data points for each siRNA were averaged and used for neural net training and testing. Inhibition data before normalization will be made available on request to the authors." This correction does not alter any of the data, the tables, the figures or the text in the remainder of the publication and supplementary material. The error has been corrected in the PDF versions of the article. 